Military Technical College Kobry Elkobbah, Cairo,

Egypt.

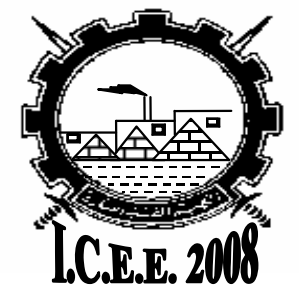

$4^{\text {th }}$ International Conference On

Chemical \& Environmental

Engineering

27-29 May 2008

\title{
CONDUCTING POLYMERS: HYDROCARBON MATERIALS THAT CONDUCT ELECTRICITY, PROSPECTIVE AND APPLICATIONS IN ENERGY CONVERSION AND SENSORS
}

\begin{abstract}
AHMED GALAL*
ABSTRACT

Polymers (or plastics as they are also called) are known to have good insulating properties. Polymers are one of the most used materials in the modern world. Their uses and application range from containers, various industry domains to clothing. For example, they are used to coat metal wires to prevent electric shocks. However it is now recognized that there are some polymers which have conducting properties. As a good example poly(acetylene) has been discovered to have these properties and can be doped to acquire unique conducting properties.

The discovery of conducting polymers opened up many new potential applications for devices combining unique optical, electrical, and mechanical properties. The literature on conducting polymers as reflected in the extensive citation of papers shows how diverse and versatile these materials can be used in different optical effects that were achieved, and underlying physical processes. This class of materials include organic polymers, copolymers, and conjugated polymers, such as poly(para-phenylene), poly(aniline), poly(hetero-arylenes) and poly(pphenylenevinylene). These can be fabricated to have high flexibility. The common devices introduced are mainly light-emitting diodes (LEDs), or, more recently, lasers, and the color of the light emitted can be chemically tuned. The main physical process involved is electroluminescence. The conducting polymers can be induced to transfer electrons to other materials such as Buckminsterfullerene. Inorganic materials can also be used to create LEDs, such as InGaN materials, or cadmium selenide nanocrystals, where the physical process involves quantum-wells.

Some of our interest lies in exploring these materials as electro-catalysts for the purpose of direct energy conversion such as anode materials in direct methanol fuel cells (DMFC). On the other hand, we are also interested in the use of conducting polymer films as modified surfaces for the application in the area of sensors.

In this presentation, we would like to introduce the audiences to some of the fundamental aspects of conducting polymeric materials as well as to snapshots of our research group latest data in the areas of electro-catalysis and sensors.
\end{abstract}

\footnotetext{
* Department of Chemistry, Faculty of Science, University of Tanta, Tanta, Egypt
} 